\title{
Primera cita de Cylindropuntia kleiniae (DC.) F. M. Knuth en la península ibérica
}

\author{
D. GUILLOT \\ c. La Pobleta, 7, ES-46118 Serra, España
}

ORCID iD: D. GUILLOT: http://orcid.org/0000-0002-1122-9762

E-mail: dguillot_36@hotmail.com

Editor: A. Susanna

Recibido 10 junio 2014; aceptado 16 junio 2016; publicado on line 5 abril 2017

\begin{abstract}
First Report of Cylindropuntia kleiniae (DC.) F. M. Knuth from the Iberian Peninsula. - Cylindropuntia kleiniae is recorded for the first time from the Iberian Peninsula.

Key words: alien flora; Cylindropuntia kleiniae; Iberian Peninsula.

\section{Resumen}

Primera Cita de CylindRopuntia KLEINIAE (DC.) F. M. KNUTH En la Península ibÉRICA. - Se cita por vez primera en la península ibérica Cylindropuntia kleiniae.

Palabras clave: Cylindropuntia kleiniae; flora alóctona; península ibérica.
\end{abstract}

\section{Cómo citar este artículo / Citation}

Guillot, D. 2017. Primera cita de Cylindropuntia kleiniae (DC.) F. M. Knuth en la península ibérica. Collectanea Botanica 36: e002. doi: http://dx.doi.org/10.3989/collectbot.2017.v36.002

\section{Copyright}

(C) 2017 CSIC. This is an open-access article distributed under the terms of the Creative Commons Attribution License (CC BY) Spain 3.0.

Se cita por primera vez como alóctona en la península ibérica la especie Cylindropuntia kleiniae (DC.) F. M. Knuth (三 Opuntia kleiniae DC.).

España, Valencia: Olocau, Pla de la Torre, UTM 30S YJ1393, $200 \mathrm{~m}$, margen de camino, en la pendiente a un barranco, 15.01.2013, D. Guillot (BC 955800). Fig. 1.
El comúnmente denominado cardoncillo, tasajillo o tasajo es una planta suculenta nativa del desierto de Chihuahua, de Nuevo México y Texas en Estados Unidos, y de los estados de Coahuila, Chihuahua, Durango y Nuevo León, en México (Anderson, 2001). En su rango de distribución natural, Cylindropuntia kleiniae coloniza pisos de jarilla-mesquite, en laderas rocosas de piedra caliza, 

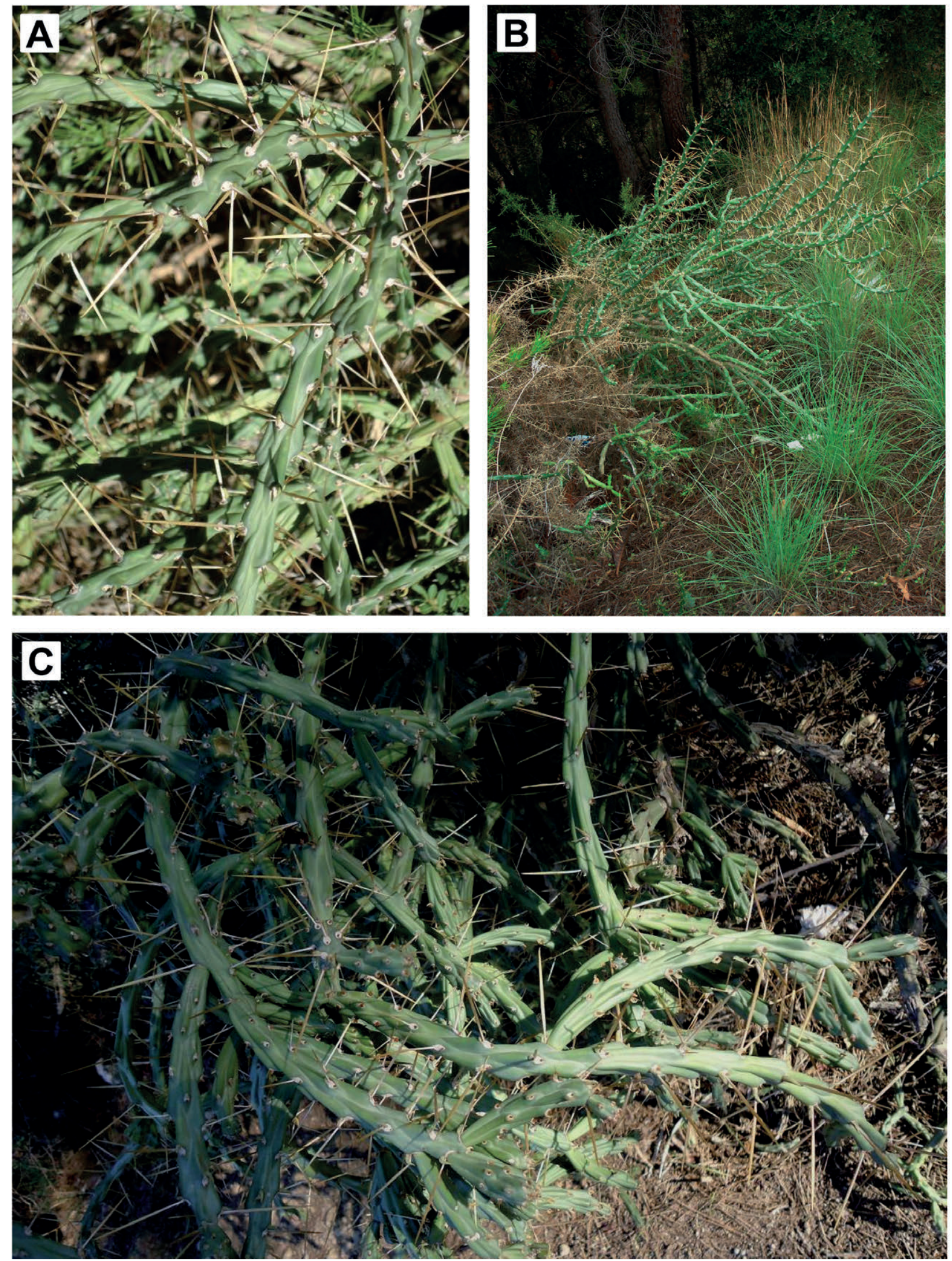

Figura 1. Cylindropuntia kleiniae en Olocau (Valencia): (A) y (C), detalle de los tallos, aréolas, glóquidas y espinas; (B), aspecto general de la planta. 
en altitudes de 800-1800 m (Pinkava, 2003). En el oeste de Texas y el norte de México, forma híbridos con C. leptocaulis (DC.) F. M. Knuth, denominados $C$. $\times$ antoniae $\mathrm{P}$. V. Heath, con grados de variación morfológica. En el norte de México, C. kleiniae hibrida con C. imbricata (Haw.) F. M. Knuth var. imbricata. De hecho, la misma Cylindropuntia kleiniae podría ser de origen híbrido (Pinkava, 2003).

Esta especie no se encuentra incluida en trabajos de catalogación de la flora alóctona valenciana (Guillot et al., 2009a; Sanz-Elorza et al., 2011), en los trabajos de catalogación de la flora cultivada en la provincia de Valencia (Guillot et al., 2009b), ni en el catálogo de la flora alóctona española (SanzElorza et al., 2004).

Según la descripción de Anderson (2001), se trata de un arbusto con ramificación abierta de 0,5-2,5 $\mathrm{m}$ de altura, con segmentos del tallo de $4-20 \mathrm{~cm}$ de longitud y $0,6-1,2 \mathrm{~cm}$ de diámetro, con tubérculos; aréolas con lana amarilla, oscureciéndose con la edad, ovales; glóquidas amarillas, de 0,5-2,5 $\mathrm{mm}$ de longitud; espinas 1-4 en la mayoría de las aréolas, en ocasiones ausentes, no oscureciendo los tallos, aciculiformes, de color amarillo a gris con ápices claros, rectas o ligeramente curvadas, de $1-3 \mathrm{~cm}$ de longitud; flores verdosas en la base, rojo o magenta en la zona superior y frutos obovoides a cilíndricos, carnosos, inermes, de color verde, pasando a rojo, de 1,3-3,4 cm de longitud y 1-2 $\mathrm{cm}$ de diámetro. Se diferencia claramente de la mayoría de táxones congenéricos citados en la península ibérica (Tabla 1) por el diámetro de los artículos $(0,6-1,2 \mathrm{~cm})$, pudiendo coincidir en este carácter con $C$. bigelovii (Engelm.) F. M. Knuth $(1-2 \mathrm{~cm})$ y $C . \times$ tetracantha (Toumey) F. M. Knuth (10-15 mm), pero difiriendo claramente de la primera, entre otros caracteres, en el número de espinas (1-4 en C. kleiniae, 6-8 en C. bigelovii) y de la segunda en caracteres como el color de las glóquidas (amarillo en C. kleiniae, marrón en $C . \times$ tetracantha) y la presencia de espinas cortas en el fruto, en $C$. $\times$ tetracantha.

\section{AGRADECIMIENTOS}

A los doctores E. Dana (Agencia de Medio Ambiente y Agua de Andalucía, División de Sostenibilidad y Medio Ambiente Urbano), P. P. Garcillán (CIBNOR, Instituto Politécnico Nacional, La Paz, B.C.S. México) y J. P. Rebman (San Diego Natural History Museum), por su ayuda para la correcta identificación de esta especie.

\section{REFERENCIAS BIBLIOGRÁFICAS}

Anderson, E. F. 2001. The cactus family. Timber Press, Portland. http://dx.doi.org/10.5962/bhl.title.46288

Britton, N. L. \& Rose, J. N. 1919. The Cactaceae. Descriptions and illustrations of plants of the cactus family 1 . The Carnegie Institution of Washington, Washington.

Guillot, D., Laguna, E. \& Rosselló, J. A. 2009a. Flora alóctona valenciana: familia Cactaceae (Monografías de la revista Bouteloua, 5). Jolube Consultor y Editor Ambiental, Jaca.

Guillot, D., Mateo, G. \& Rosselló, J. A. 2009b. Claves para la flora ornamental de la provincia de Valencia (Monografías de la revista Bouteloua, 1). Jolube Consultor y Editor Ambiental, Jaca.

Pinkava, D. J. 2003. Cylindropuntia. In: Flora of North America Editorial Committee (Eds.), Flora of North America North of Mexico 4. Oxford University Press, New York \& Oxford: 103-118.

Sanz-Elorza, M., Dana, E. D. \& Sobrino, E. 2004. Atlas de las plantas alóctonas invasoras en España. Dirección General para la Biodiversidad, Madrid.

Sanz-Elorza, M., Guillot, D. \& Deltoro, V. 2011. La flora alóctona de la Comunidad Valenciana (España). Botanica Complutensis 35: 97-130. http://dx.doi.org/10.5209/rev_ BOCM.2011.v35.10 


\begin{tabular}{|c|c|c|c|c|c|c|c|c|}
\hline 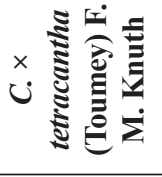 & 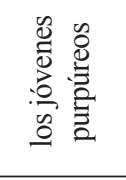 & 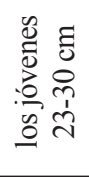 & 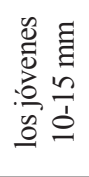 & | & 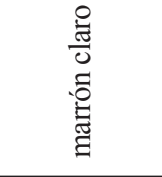 & 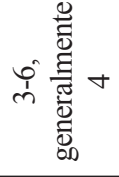 & & 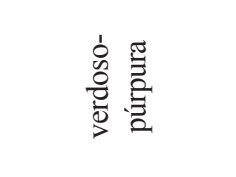 \\
\hline 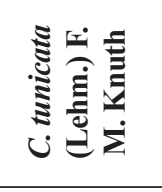 & 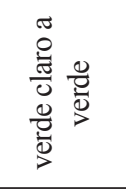 & 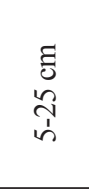 & $\begin{array}{l}\tilde{0} \\
n \\
n \\
i \\
\text { ñ } \\
-\end{array}$ & 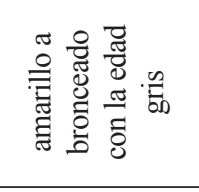 & 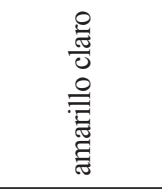 & $\stackrel{7}{n}$ & 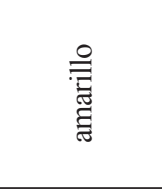 & 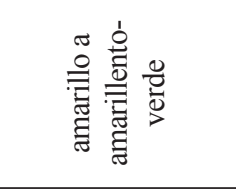 \\
\hline 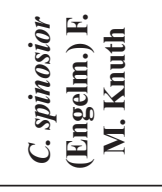 & 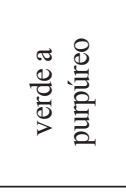 & $\begin{array}{l}\text { E } \\
\overparen{1} \\
i \\
i n\end{array}$ & 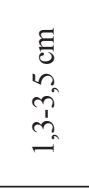 & 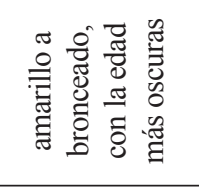 & 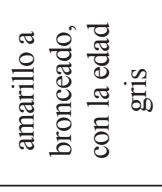 & 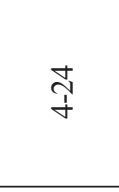 & 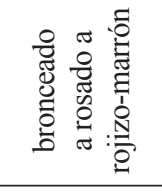 & 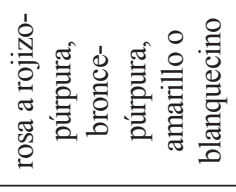 \\
\hline 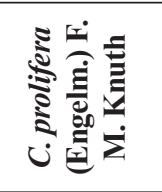 & 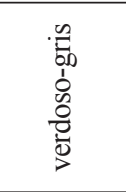 & $\begin{array}{l}\bar{y} \\
\frac{n}{7}\end{array}$ & $\begin{array}{l}\tilde{y} \\
n \\
n \\
n \\
n\end{array}$ & 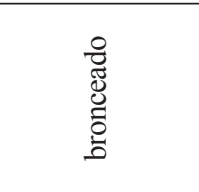 & 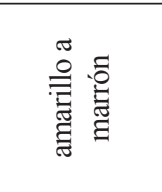 & $\frac{7}{b}$ & 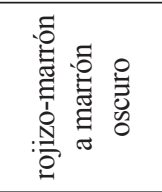 & 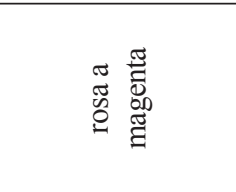 \\
\hline 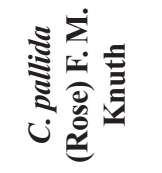 & 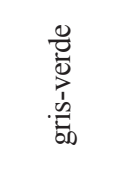 & $\begin{array}{l}\tilde{y} \\
n \\
\vdots \\
0 \\
0\end{array}$ & $\begin{array}{l}\text { E } \\
n \\
\hat{n} \\
\text { b. } \\
-1\end{array}$ & $\mid$ & 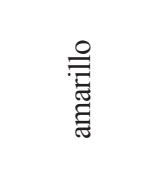 & 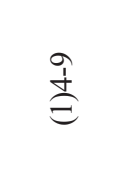 & 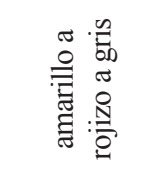 & $\underset{\mathscr{d}}{\mathscr{b}}$ \\
\hline 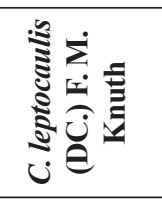 & 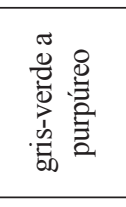 & $\begin{array}{l}\text { च } \\
0 \\
\infty \\
\text { ஸे }\end{array}$ & 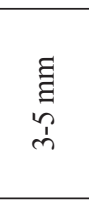 & 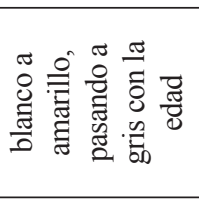 & 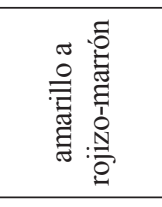 & $\frac{\widehat{O}}{\frac{1}{0}}$ & 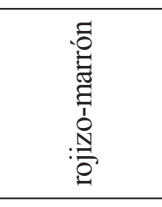 & 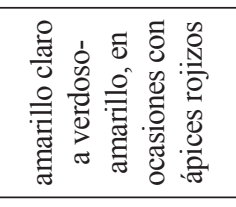 \\
\hline 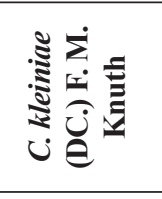 & $\mid$ & $\begin{array}{l}\tilde{\Xi} \\
\stackrel{\leftrightarrow}{7} \\
\text { t }\end{array}$ & 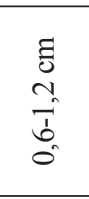 & 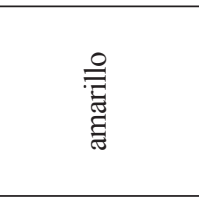 & $\begin{array}{l}\text { 䔍 } \\
\text { 䒕 }\end{array}$ & \pm & 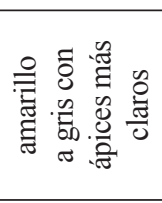 & 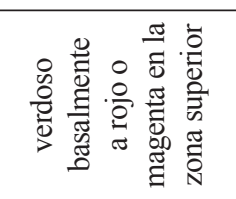 \\
\hline 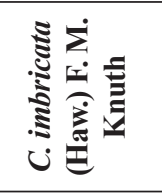 & 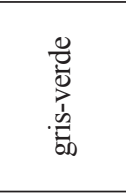 & 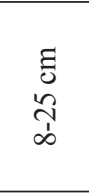 & 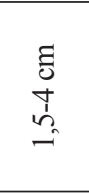 & 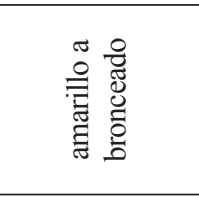 & 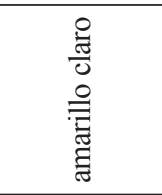 & 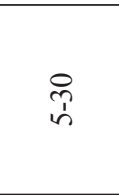 & 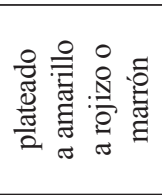 & 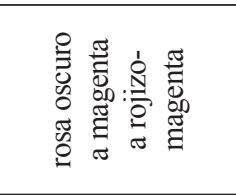 \\
\hline 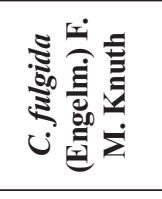 & 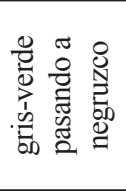 & 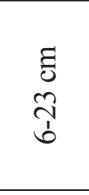 & $\begin{array}{l}\tilde{0} \\
n \\
\text { ?े } \\
\text { ते }\end{array}$ & 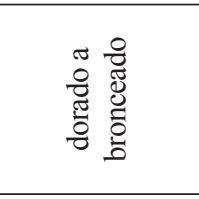 & 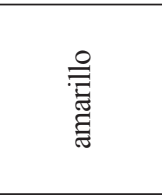 & $\frac{\infty}{b}$ & 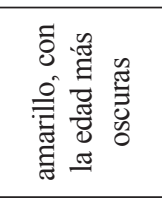 & 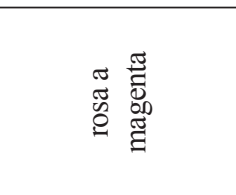 \\
\hline 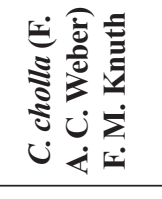 & 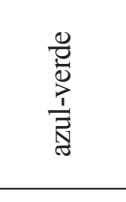 & $\begin{array}{l}\text { हี } \\
\overrightarrow{=}\end{array}$ & $\begin{array}{l}\tilde{0} \\
n \\
n \\
n \\
n\end{array}$ & 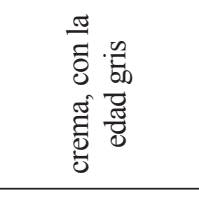 & $\begin{array}{l}\mathscr{z} \\
\overparen{\tilde{z}} \\
0 \\
0\end{array}$ & $\frac{0}{n}$ & 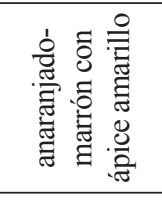 & 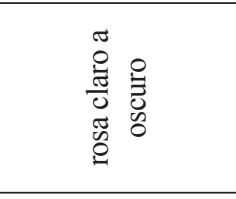 \\
\hline 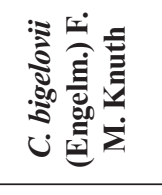 & 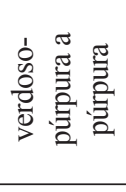 & $\begin{array}{l}\tilde{\Xi} \\
\infty \\
\frac{\infty}{f}\end{array}$ & $\begin{array}{l}\text { I } \\
\text { I }\end{array}$ & 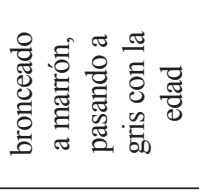 & $\begin{array}{l}\text { 号 } \\
\text { 言 } \\
\text { 离 }\end{array}$ & $\hat{b}_{b}^{\infty}$ & 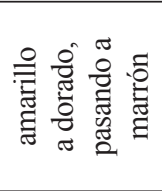 & 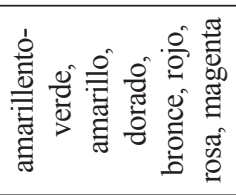 \\
\hline & 旁竞 & 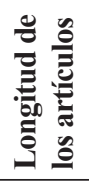 & 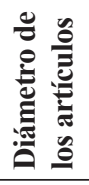 & 产 & 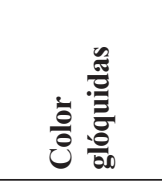 & 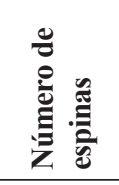 & 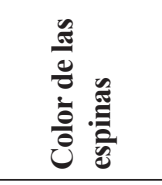 & 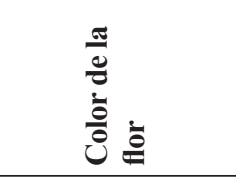 \\
\hline
\end{tabular}




\begin{tabular}{|c|c|c|c|c|c|}
\hline 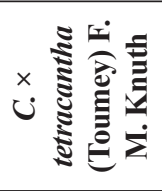 & 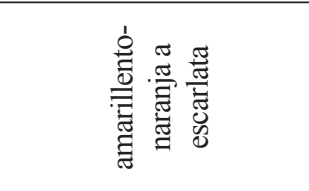 & 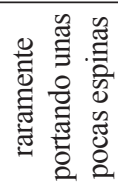 & 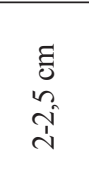 & | & $\begin{array}{l}\tilde{D} \\
\text { n. } \\
\tilde{i} \\
\text { in }\end{array}$ \\
\hline 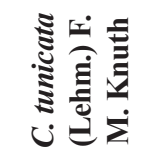 & $\stackrel{\circ}{\circ}$ & 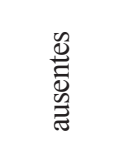 & $\mid$ & 1 & $\begin{array}{l}\text { Jే } \\
\text { o } \\
\text { ஸे }\end{array}$ \\
\hline 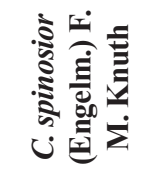 & 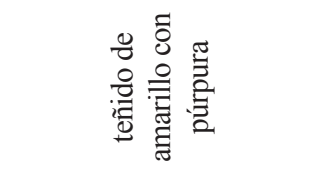 & $\mid$ & $\begin{array}{l}\Xi \\
\vdots \\
\sim \\
i\end{array}$ & 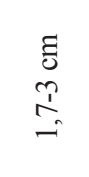 & | \\
\hline 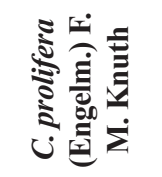 & 1 & | & 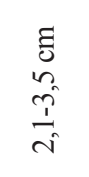 & $\begin{array}{l}\tilde{D} \\
\sim \\
\tilde{d} \\
\tilde{d}\end{array}$ & 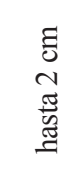 \\
\hline 离离 & $\begin{array}{l}\stackrel{\varrho}{\overline{\bar{E}}} \\
\text { हूँ }\end{array}$ & 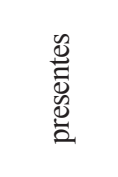 & $\begin{array}{c}E \\
0 \\
\infty \\
\vec{b} \\
\stackrel{0}{-1}\end{array}$ & 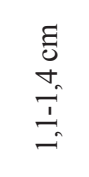 & $\begin{array}{l}\Xi \\
\pm \\
\pm\end{array}$ \\
\hline 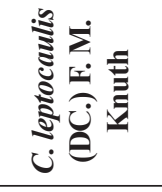 & 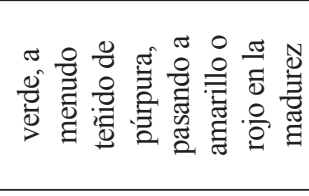 & 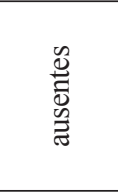 & $\begin{array}{l}\text { E } \\
\vdots \\
n \\
0 \\
0 \\
0\end{array}$ & $\frac{\Xi}{\mathfrak{g}}$ & \begin{tabular}{l} 
E \\
$n$ \\
\multirow{1}{n}{} \\
$n$ \\
$n$
\end{tabular} \\
\hline 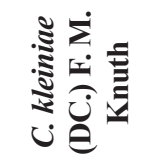 & 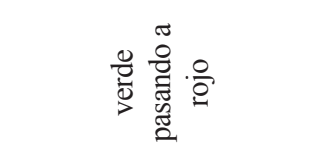 & 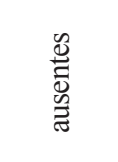 & 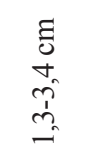 & $\begin{array}{l}\Xi \\
\\
\stackrel{1}{I}\end{array}$ & $\begin{array}{l}\tilde{0} \\
\stackrel{1}{I}\end{array}$ \\
\hline 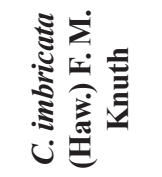 & $\begin{array}{l}\text { 을 } \\
\text { 壱 }\end{array}$ & 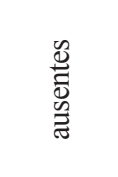 & $\begin{array}{l}\tilde{\Xi} \\
i n \\
\stackrel{+}{+} \\
\stackrel{i}{i}\end{array}$ & $\begin{array}{l}\Xi \\
0 \\
\stackrel{+}{~}\end{array}$ & | \\
\hline 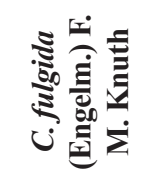 & 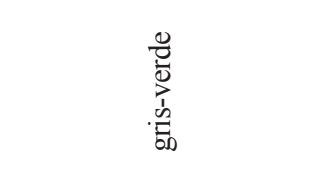 & 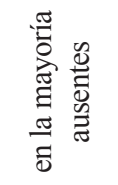 & $\begin{array}{l}\text { ह } \\
n \\
n \\
n \\
i\end{array}$ & $\begin{array}{l}\Xi \\
0 \\
n \\
\stackrel{2}{2} \\
\stackrel{2}{-}\end{array}$ & $\begin{array}{l}\tilde{y} \\
n \\
n \\
\tilde{J} \\
\tilde{J} \\
\tilde{g}\end{array}$ \\
\hline 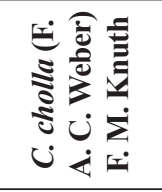 & $\frac{\mathscr{D}}{D}$ & 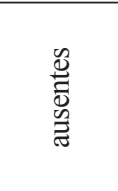 & 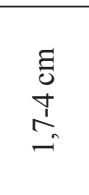 & 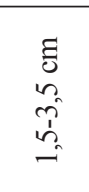 & $\begin{array}{l}\tilde{\Xi} \\
\text { Jे } \\
\stackrel{+}{+}\end{array}$ \\
\hline \multirow[t]{2}{*}{ 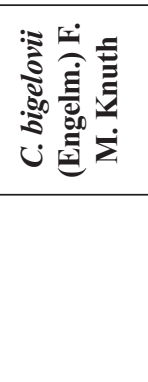 } & 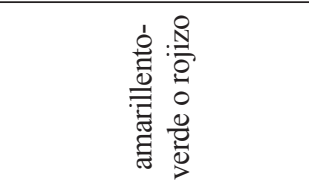 & 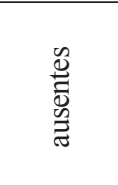 & 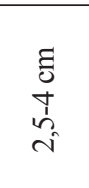 & 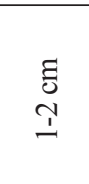 & 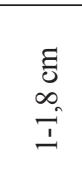 \\
\hline & $\begin{array}{l}\overline{\mathrm{g}} \\
\dot{\bar{g}} \\
\mathrm{\partial}\end{array}$ & 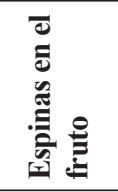 & 量 & 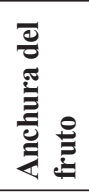 & 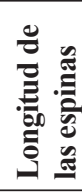 \\
\hline
\end{tabular}

